\title{
Pump-probe and pump-deplete-probe spectroscopies on carotenoids with $N=9-15$ conjugated bonds
}

Cite as: J. Chem. Phys. 125, 194505 (2006); https://doi.org/10.1063/1.2388274

Submitted: 02 June 2006. Accepted: 13 October 2006 . Published Online: 16 November 2006

Tiago Buckup, Janne Savolainen, Wendel Wohlleben, Jennifer L. Herek, Hideki Hashimoto, Ricardo R. B. Correia, and Marcus Motzkus

\section{ARTICLES YOU MAY BE INTERESTED IN}

The dependence of the ultrafast relaxation kinetics of the $S_{2}$ and $S_{1}$ states in $\beta$-carotene homologs and lycopene on conjugation length studied by femtosecond time-resolved absorption and Kerr-gate fluorescence spectroscopies

The Journal of Chemical Physics 130, 214506 (2009); https://doi.org/10.1063/1.3147008

Photophysics and dynamics of the lowest excited singlet state in long substituted polyenes with implications to the very long-chain limit

The Journal of Chemical Physics 103, 2509 (1995); https://doi.org/10.1063/1.469672

Ultrafast transient absorption revisited: Phase-flips, spectral fingers, and other dynamical features

The Journal of Chemical Physics 144, 175102 (2016); https://doi.org/10.1063/1.4947568

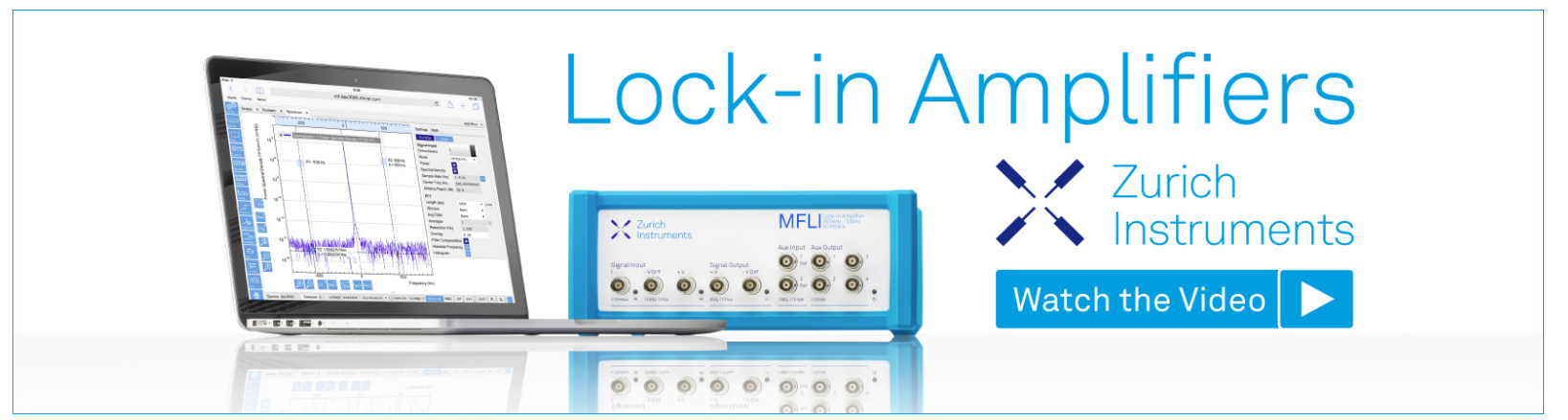

J. Chem. Phys. 125, 194505 (2006); https://doi.org/10.1063/1.2388274

(c) 2006 American Institute of Physics. 


\title{
Pump-probe and pump-deplete-probe spectroscopies on carotenoids with $N=9-15$ conjugated bonds
}

\author{
Tiago Buckup \\ Physikalische Chemie, Philipps Universität, D-35032 Marburg, Germany \\ and Instituto de Física, UFRGS, CEP 91501-970 Porto Alegre, Brazil \\ Janne Savolainen \\ FOM Institute for Atomic and Molecular Physics, 1098 SJ Amsterdam, The Netherlands \\ Wendel Wohlleben \\ BASF Aktiengesellschaft, Polymer Research Physics, D-67056 Ludwigshafen, Germany \\ Jennifer L. Herek \\ FOM Institute for Atomic and Molecular Physics, 1098 SJ Amsterdam, The Netherlands \\ Hideki Hashimoto \\ Department of Physics, Osaka University, Osaka 558-8585, Japan
}

Ricardo R. B. Correia

Instituto de Física, UFRGS, CEP 91501-970 Porto Alegre, Brazil

Marcus Motzkus ${ }^{\text {a) }}$

Physikalische Chemie, Philipps Universität, D-35032 Marburg, Germany

(Received 2 June 2006; accepted 13 October 2006; published online 16 November 2006)

\begin{abstract}
A series of all-trans-carotenoids with $N=9,13$, and 15 conjugated bonds has been studied by pump-probe and pump-deplete-probe spectroscopies to obtain a systematic analysis of the energy flow between the different electronic states. The ultrafast dynamics in the carotenoids are initialized by excitation to the $S_{2}$ state and subsequently manipulated by an additional depletion pulse in the near-IR spectral range. The changes in the dynamics after depletion of the excited state population allowed differentiation of the excited state absorption into two components, a major one corresponding to the well known $S_{1}$ state and the small contribution on the red wing of the $S_{0}-S_{2}$ absorption band originating from the hot ground state. We found no evidence for an additional electronically excited state, usually called $S^{*}$. Instead, a deactivation mechanism that includes the hot ground state supports the observed results nicely in the framework of a simple three state model $\left(S_{2}, S_{1}\right.$, and $\left.S_{0}\right)$. @ 2006 American Institute of Physics. [DOI: 10.1063/1.2388274]
\end{abstract}

\section{INTRODUCTION}

Carotenoids perform a variety of critical functions in nature including acting as photoprotection chromophores, structural elements, and accessory pigments in the light harvesting. ${ }^{1}$ Their photochemistry depends strongly on the deactivation dynamics of the first two excited low-lying singlet states. ${ }^{2}$ In the current standard energy model for carotenoids, the excitation by a blue-green photon in the visible region is to the $S_{2}$ state $\left(1 B_{u}^{+}\right)$, the first allowed one-photon transition from the ground state $S_{0}\left(1 A_{g}^{-}\right)$. The population then decays through two channels: fast internal conversion to the lower-lying singlet excited state $S_{1}\left(2 A_{g}^{-}\right)$or directly back to the ground state emitting fluorescence. ${ }^{3}$ Furthermore, as one could expect, in the internal conversion between $S_{2}$ $-S_{1}$ the excited vibrational levels of $S_{1}$ take part in the deactivation. ${ }^{4-6}$ The $S_{1}$ state decays much slower to the ground state with time constants lying in the picosecond time scale and strongly depends on the length of the carotenoid, ${ }^{5}$ as satisfactorily described by the energy gap rule. ${ }^{7}$ This

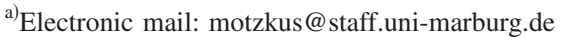

simple three level model was challenged by theoretical calculations of Tavan and Schulten who predicted the presence of two or three additional dark states lying in the vicinity of the $S_{2}$ state, which could also be active in the deactivation network. Some experimental work supports this claim, for example, the $1 B_{u}^{-}$and $3 A_{g}^{-}$states have been tentatively identified in transient absorption measurements performed in the near IR (Ref. 8) and more recently in the visible, ${ }^{9}$ where depending on the carotenoid length, either the $3 A_{g}^{-}$or the $1 B_{u}^{-}$ would take part in the internal conversion. Besides that, evidences for these states were also found in static resonant Raman excitation profiles, in agreement with the predicted energy scaling. ${ }^{10-12}$ Measurements performed for beta carotene in the near IR with high temporal resolution attempt to associate a sub-50 fs dynamics around $800-1000 \mathrm{~nm}$ to the $1 B_{u}^{-}$dark state (though for lack of certainly label it simply $\left.S_{x}\right){ }^{13}$ This result was recently shown to be the outcome of a two-photon absorption to a higher singlet state. ${ }^{14}$ In another set of experiments, the $1 B_{u}^{-}$has been associated with absorption on the red wing of the $S_{0}-S_{2}$ absorption band (usually called $S^{*}$ ) in light harvesting complexes, ${ }^{15,16}$ while the same wing is explained as a vibrationally excited ground state (hot 

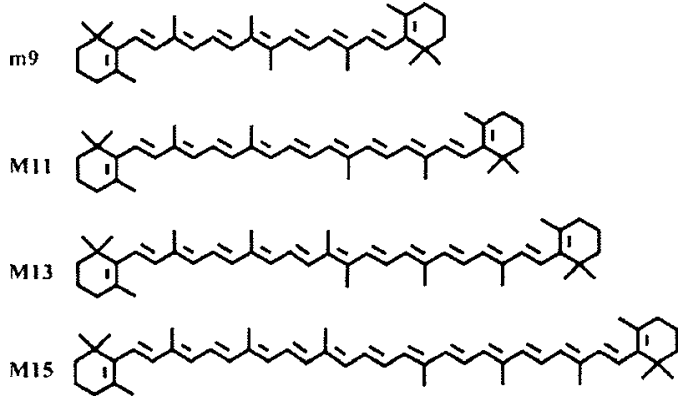

FIG. 1. Chemical structure of $\beta$-carotene homologs. The uppercase $\mathrm{M}$ stands for Macro- $\beta$-carotene and the lowercase for mini- $\beta$-carotene. The numbers stand for the number of conjugated double bonds.

$S_{0}$ ) in long carotenoids. ${ }^{17}$ In the latter study, the main argument was based at the very different decay times between $S_{1}$ and $S^{*}$ measured for carotenoids with 15 and 19 conjugated bonds. In the case of $N=15$, decays of 1.1 and $6.6 \mathrm{ps}$ were encountered for $S_{1}$ and $S^{*}$, respectively. Very recently, Papagianakis et al. ${ }^{18}$ reinvestigated the carotenoid (rhodopin glucoside) embedded in the light harvesting complex (R.acidophila) and proposed a mechanism for the origin of the redwing contribution of the $S_{0}-S_{2}$ absorption band by means of the relaxation of a highly lying $S_{n}$, which is excited via a two-photon excitation of the pump pulse. In order to obtain reasonable strong transition probabilities the two-photon excitation model postulates the existence of an excited state absorption (ESA) transition in the visible from $S_{2}$. Although the environment of the protein matrix probably leads here to different conformations of the carotenoid and therefore opens additional relaxation channels, the presence of nonlinear interactions is an important aspect which should be always considered since their influence is often underestimated. ${ }^{14}$

From the results cited above it is obvious that all these proposed experimental observations do not agree in every aspect and further analysis is needed to achieve a generalized picture.

In order to help to disentangle the nature of these spectral signatures, we addressed the question whether these intermediary dark states are playing any role in the deactivation network of carotenoids in solution and what is the nature of $S^{*}$ by using a modified pump-probe technique. ${ }^{19}$ The obtained results led us to conclude that the assumption of a hot$S_{0}$ component can be associated with the red wing of the $S_{0}-S_{2}$ absorption band and therefore the energy level scheme with three different electronic states, $1 B_{u}^{+}, 2 A_{g}^{-}$, and $1 A_{g}^{-}$, describes satisfactorily the entire dynamical behavior.

In this paper, a series of all-trans-carotenoids with $N$ =9 -15 conjugated bonds (labeled $\mathrm{m} 9, \mathrm{M} 11, \mathrm{M} 13$, and M15; see Fig. 1) was studied in order to further investigate the hot$S_{0}$ hypothesis. First, transient absorption measurements were done systematically for this family of carotenoids with similar structure and terminal groups, focusing on the comparison of the relaxation times of the red wing of the $S_{0}-S_{2}$ absorption band, where the $S^{*}$ band should be located, with the $S_{1}$ transient absorption peak. In a second experiment, the pump-depletion-probe experiment was performed using a higher temporal resolution (almost three times better) than in our previous work ${ }^{19}$ and also varying the wavelength of the depletion pulse. The idea behind this three pulse pump-probe technique is to launch the system to an excited state and then interfere with the evolution of the system at a point which is normally inaccessible with just one pump pulse. ${ }^{19-22}$ In the case of carotenoids, this point is exactly the moment that follows the arrival of population at $S_{2}$, thus depleting the usual decay pathway. We show that the interpretation of the red wing of the $S_{0}-S_{2}$ absorption band being a vibrationally excited ground state, instantaneously populated by stimulated Raman scattering, is again consistent with the experimental results.

\section{EXPERIMENTAL DETAILS}

The all-trans-carotenes $\mathrm{m} 9, \mathrm{M} 13$, and M15 were synthesized by a reduction dimerization (McMurry reaction) as described in Ref. 5. The all-trans- $\beta$-carotene (M11) was purchased from Aldrich and used as received. All samples were prepared in $\mathrm{N}_{2}$ atmosphere, in order to avoid degradation. They were dissolved in benzene without any further purification, resulting in a maximum static absorption of the $S_{0}-S_{2}$ transition between 0.25 and 0.4 optical density (OD). A rotating cell with windows of $1 \mathrm{~mm}$ thickness was used in all experiments.

The experimental configuration of the pump-probe experiment was very similar to Ref. 19. The pump laser pulse was provided by a homebuilt noncollinear optical parametric amplifier (nc-OPA) which was varied between 480 and $560 \mathrm{~nm}$ in the wavelength dependence studies. Pulse durations were below 35 fs. White-light continuum was used as probe pulse in all experiments which was generated in a sapphire crystal by a fraction of the energy of the fundamental laser $(800 \mathrm{~nm})$. In the transient absorption experiments the pump was tuned to the $S_{2} 0-0$ vibrational band for carotenoids with $N \geqslant 11: 495,525$, and $555 \mathrm{~nm}$ for M11, M13, and M15, respectively. In the case of $\mathrm{m} 9$, the idler of the nc-OPA at $900 \mathrm{~nm}$ was doubled to $450 \mathrm{~nm}$ and used to pump $\mathrm{m} 9$ between the $0-0$ and the $0-1$ peak.

The main difference between this setup and our previous experiment ${ }^{19}$ was the use of a second homebuilt nc-OPA in the depletion experiments, which gave a much higher temporal resolution to the depletion experiments. Depletion measurements were made using two distinct wavelengths, 940 and $1000 \mathrm{~nm}$. In both cases, the spectrum had a full width at half maximum (FWHM) around $50 \mathrm{~nm}$ and pulse duration below 40 fs. The detection system was improved by the use of a diode array in combination with a spectrograph (Acton 300i).

In order to find precisely the zero delay between the visible pump pulse and near-IR (NIR) depletion beam, a characterization setup was built to measure the cross correlation (XC) at the exact position of the sample. The zero delay between the white light and pump laser was determined using the nonresonant coherent artifact in pure benzene generated in the same cell. The zero-delay point could be determined with an error smaller than $10 \mathrm{fs}$. The energies used for the pump were less than $80 \mathrm{~nJ}$ and for the depletion 

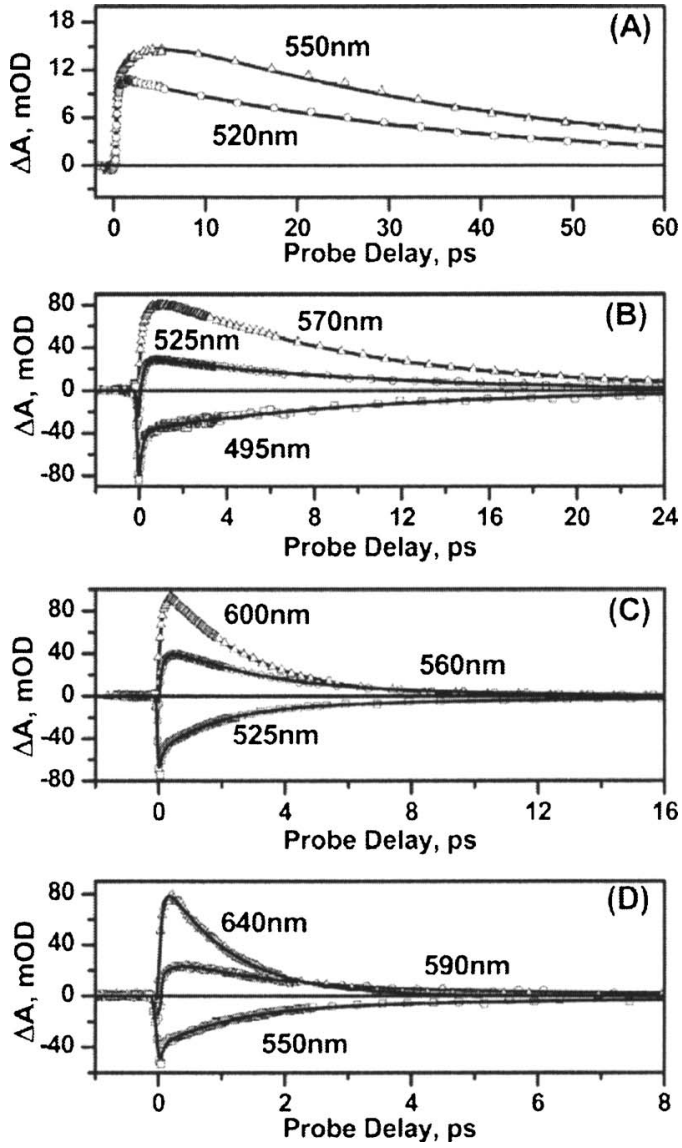

FIG. 2. Fitted pump-probe transients for (A) m9, (B) M11, (C) M13, and (D) M15 at different wavelengths corresponding to induced absorption from $S^{*}$ (circles) and $S_{1}$ (triangles) and ground state bleach (squares). For all carotenoids, the bleach signal corresponds to the shortest wavelength, the $S$ signal is at the intermediate wavelength and the $S_{1}$ signal is the reddest.

less than $1.4 \mu \mathrm{J}$. After focusing with concave mirrors the intensities were around $4 \times 10^{14}$ and $5 \times 10^{15}$ photons $/ \mathrm{cm}^{2}$, respectively.

\section{PUMP-PROBE-MEASUREMENTS}

The kinetic transients of all four studied carotenoids are shown in Fig. 2. The excitation always took place at the 0-0 transition leading to continuously increasing wavelengths for the respective peaks of $S_{0}-S_{2}$ bleaching, and $S^{*}$ and $S_{1}$ bands. Note here that the label $S^{*}$ refers simply to the excited state absorption band appearing on the red edge of $S_{0}-S_{2}$ absorption band and not as a separate excited state as previously defined in Ref. 16. The results are summarized in the Table I. In many aspects, all kinetics show similar behavior.

\section{A. Rise time of $S_{1}$ and $S^{\star}$}

The rises of the $S_{1}$ signals were fitted with two exponential components in all carotenoids when pumped at $0-0$. The slower component is associated with the decay time of the $S_{1}$ precursor, a hot $S_{1}$, and it ranges from $0.55 \pm 0.10 \mathrm{ps}(9 \%$ contribution) in the M15 to $3.6 \pm 0.4 \mathrm{ps}(40 \%)$ in $\mathrm{m} 9$. These values are in good agreement with previous studies. ${ }^{5,19}$ Also the shorter time component for M11, 170 $\pm 10 \mathrm{fs}$, matches well with other measurements. ${ }^{23}$ For $\mathrm{m} 9$, this second component does not increase substantially and is around $180 \pm 5 \mathrm{fs}$. The values of the second component for M13 and M15 are both below the $100 \mathrm{fs}$ time scale, $90 \pm 10$ and $50 \pm 10 \mathrm{fs}$, respectively, and are in line with the results from a previous experiment. $^{24}$

The rise of the $S^{*}$ signal exhibits a more interesting behavior but is more complicated to fit due to the overlap with the stimulated emission from $S_{2}$. For the smaller molecules $\mathrm{m} 9$ and M11, the rise time was fitted with single exponentials of $190 \pm 10$ and $140 \pm 10 \mathrm{fs}$, respectively. However, for the longer carotenoids, M13 and M15, the growth rates had to be fitted with two time constants. One of them is very fast and could not be resolved by our experimental setup, while the slower contribution lies in the same range as the fast component of the $S_{1}$ rise, 50 and $90 \mathrm{fs}$, respectively. The rise time measured for all homologs matches the fluorescence lifetime for $S_{2}$ found recently in other studies. ${ }^{23,25}$ Particularly, the femtosecond fluorescence experiment of Konsumi et $a .^{25}$ shows a remarkable agreement with our rise times: 70 fs for M15, 90 fs for M13' (absence of methyl group), $150 \mathrm{fs}$ for M11, and $210 \mathrm{fs}$ for $\mathrm{m} 9$.

From the data for the rise times of the $S_{1}$ and $S^{*}$ bands it can be concluded so far that the relaxation path towards $S_{1}$

TABLE I. Relaxation and rise time constants for the carotenoid homologs. Fluorescence times, when available, are also shown. All values in ps.

\begin{tabular}{ccccc}
\hline \hline & $\mathrm{m} 9$ & M11 & M13 & M15 \\
\hline$S_{1}$ & $41.5 \pm 0.9^{\mathrm{a}}$ & $9.5 \pm 0.2^{\mathrm{a}}$ & $2.52 \pm 0.02^{\mathrm{a}}$ & $1.02 \pm 0.02^{\mathrm{a}}$ \\
& & $9.3 \pm 0.4^{\mathrm{b}}$ & $2.8^{\mathrm{c}}$ & $1.1^{\mathrm{c}}$ \\
$S^{*}$ & $39.1 \pm 0.9^{\mathrm{a}}$ & $10.2 \pm 0.1^{\mathrm{a}}$ & $6.7 \pm 0.5^{\mathrm{a}}$ & $7.7 \pm 0.9^{\mathrm{a}}$ \\
Bleach recovery & NA & (I) $0.16 \pm 0.02(65 \%)^{\mathrm{a}}$ & $\left(\right.$ I) $0.17 \pm 0.02(79 \%)^{\mathrm{a}}$ & (I) $0.15 \pm 0.02(81 \%)^{\mathrm{a}}$ \\
(two components) & & (II) $10.4 \pm 0.2(35 \%)^{\mathrm{a}}$ & (II) $7.4 \pm 0.9(21 \%)^{\mathrm{a}}$ & $\left(\right.$ II) $8 \pm 1(19 \%)^{\mathrm{a}}$ \\
$S_{2}$ & $0.180 \pm 5$ & $0.17 \pm 0.1^{\mathrm{a}}$ & $0.09 \pm 0.01^{\mathrm{a}}$ & $0.05 \pm 0.01^{\mathrm{a}}$ \\
& & $0.16 \pm 0.01^{\mathrm{e}}$ & $0.112 \pm 0.003^{\mathrm{e}}$ & $0.047 \pm 0.05^{\mathrm{e}}$ \\
Fluorescence & $0.210^{\mathrm{d}}$ & $0.180 \pm 0.015^{\mathrm{f}}, 0.150^{\mathrm{d}}$ & $0.09^{\mathrm{d}}$ & $<0.1^{\mathrm{g}}, 0.070^{\mathrm{d}}$ \\
\hline \hline
\end{tabular}

${ }^{\mathrm{a}}$ This work.

${ }^{\mathrm{b}}$ Reference 19.

${ }^{c}$ Reference 29.

${ }^{\mathrm{d}}$ Reference 25 .

${ }^{\mathrm{e}}$ Reference 24.

${ }^{\mathrm{f}}$ Reference 26.

${ }^{\mathrm{g}}$ Reference 17. 
necessitates a hot $S_{1}$ state and that the rise time of the $S^{*}$ corresponds roughly to the $S_{2}$ lifetime. The spectral overlap between the $S^{*}$ and the stimulated emission does not allow an unambiguously analysis of the rise time of the $S^{*}$ signal, i.e., whether it rises instantaneously or with a finite rise time. ${ }^{19}$ The assumption of an intermediate state between $S_{2}$ and $S_{1}$ is not supported but also cannot be completely abandoned because of our temporal resolution. In principle, it is possible that the faster rise component observed for all carotenoid $S_{1}$ states could be hiding an intermediate dark state.

\section{B. Decay time of $S_{1}$ and $S^{\star}$}

The decay of the peak of the $S_{1}$ ESA band was fitted with just one decay rate for all molecules and pump wavelengths. As expected from the energy gap rule, the longer the carotenoid, the faster is the decay: $\mathrm{m} 9$ with $41.5 \pm 0.6 \mathrm{ps}$, M11 with $9.5 \pm 0.2 \mathrm{ps}, \mathrm{M} 13$ with $2.5 \pm 0.1 \mathrm{ps}$, and M15 with $1.02 \pm 0.02 \mathrm{ps}$. These values are again in good agreement with the ones obtained by other groups. ${ }^{5,17,23,24}$ The decay of $S^{*}$ shows a different behavior instead. For $\mathrm{m} 9$ and M11, the $S^{*}$ decay seems to correspond to a single exponential decay with a time constant of $S_{1}$, independent of the excitation wavelength, e.g., if the molecule was pumped at $0-0$ or higher. For M13 and M15, it was necessary to fit the decay with two components. The faster component scales with the single decay of $S_{1}, 1.05$ and $2.5 \mathrm{ps}$, respectively, while the slower one is $6.7 \pm 0.5 \mathrm{ps}(50 \%)$ for M13 and $7.7 \pm 0.9 \mathrm{ps}$ (22\%) for the M15. These values of the $S^{*}$ decay are very different from the $S_{1}$ decay values for these two molecules. A similar behavior has been also observed in Ref. 17 for the same wavelength in M15 but for M13, no data are available. The faster component of $S^{*}$ band might be explained by contribution from $S_{1}$ since the broad bands lead to considerable overlap at this spectral position. The fact that $S^{*}$ decays, for carotenoids with $N \leqslant 11$, with a similar time constant of $S_{1}$, while for longer ones it has a slower decay, is a strong indication that $S_{1}$ and $S^{*}$ have a different nature. The decay time of $S^{*}$ does not vary as $S_{1}$ with the carotenoid length, i.e., it does not obey the exponential energy gap rule like $S_{1}$ does. The $S^{*}$ lifetime dependence on the number of double bonds and the spectral superposition between the $S^{*}$ band and the fluorescence of $S_{2}$ lead us to propose that $S^{*}$ is a vibrationally excited ground state. The decay of $S^{*}$ is then the cooling of the hot $S_{0}$ to the cold $S_{0}$. This hypothesis will be later reinforced by the results from the depletion experiment.

\section{Bleach of $S_{2}-S_{0}$ transition}

For carotenoids with $N \geqslant 11$, it was additionally possible to measure the bleach signal at shorter wavelengths at $495 \mathrm{~nm}$ (M11), $525 \mathrm{~nm}$ (M13), and $550 \mathrm{~nm} \mathrm{(M15)} \mathrm{which}$ can be attributed to transitions between the ground state $S_{0}$ and the $S_{2}$ state. In the case of M11 which has been also investigated in our previous work, it is not possible to relate the origin of the bleach recovery to $S^{*}$ or $S_{1}$ because of the similar values of the time constants. However, in the case of the two longer carotenoids, M13 and M15, the slower component is unambiguously in the same range of the $S^{*}$ decay.
The strong correlation adds another argument that supports the existence of a population deactivation pathway that is different from $S_{1}$.

\section{Dependence of dynamics on excitation wavelength}

Transient absorption measurements were also done for different pump wavelengths in order to study the influence of vibrationally excited levels in $S_{2}$ on the energy flow. The study was performed on M15 only since it exhibits the biggest difference between $S^{*}$ and $S_{1}$. The pump laser was tuned from the $S_{2}$ 0-0 transition to the 0-1 and 0-2 bands for this purpose. Two observations can be made which show small differences from the general behavior of the transients: a long component in the $S_{1}$ spectral region and an alteration of the $S_{1}$ rise time. Because of the small contribution $(<4 \%)$ of the additional decay component, it is difficult to extract very accurate numbers from the fit. The component is only observable in the $S^{*}$ region and it is restricted to the center blue wing region of the ESA. The change of the excitation wavelength to the 0-2 transition leads in the case of the $S_{1}$ rise time to a slower time constant ( $80 \mathrm{fs}$ ) compared to the excitation of the 0-0 transition (50 fs). Such behavior is expected for the propagation of a wave packet on the $S_{2}$ surface: the higher the wave packet is launched on the $S_{2}$ surface, the longer it takes for the wave packet to evolve on the $S_{2}$ potential energy surface and consequently delaying the population relaxation. Such vibrational cooling inside $S_{2}$ was already observed for smaller carotenoids (neurosporene) ${ }^{26}$ but nothing similar has been observed for the $S^{*}$ band.

\section{PUMP-DEPLETE-PROBE MEASUREMENTS}

The depletion experiment makes use of the fast $S_{2}-S_{n}$ IR ESA bands recently observed for several carotenoids. ${ }^{8,13,24} \mathrm{It}$ consists of keeping the delay between the pump and probe constant and then scanning the delay between an additional depletion pulse and the fixed pump-probe pulses. The idea of this scheme is to change the population after the excitation by intercepting the normal relaxation by depletion and to identify specific states or nodes of the energy flow network which are dependent on this additional pulse. The depletion experiments were made for M11, M13, and M15. The results are summarized in Fig. 3 and lead to a consistent behavior independent of the molecule and of the pump or depletion pulse wavelength: the depleted band is centered at the $S_{1}$ absorption band, while the $S^{*}$ band is not affected at all by the depletion pulse [Figs. 3(A), 3(C), and 3(E)]. Further information can be obtained by scanning the depletion pulse while keeping the pump and probe pulses fixed in time. These curves are called depletion action traces because they measure the amplitude of the depletion effect against the delay between the pump and the depletion pulses.

The depletion action trace [Figs. 3(B), 3(D), and 3(F)] was fitted with one single exponential for the rise and two exponentials for the decay. The rise of the depletion action trace was always faster than our experimental resolution yielding values below 25 fs. The recovery of the depletion action trace shows a more complex dynamics which depend also on the specific molecule. It consists of two components, 

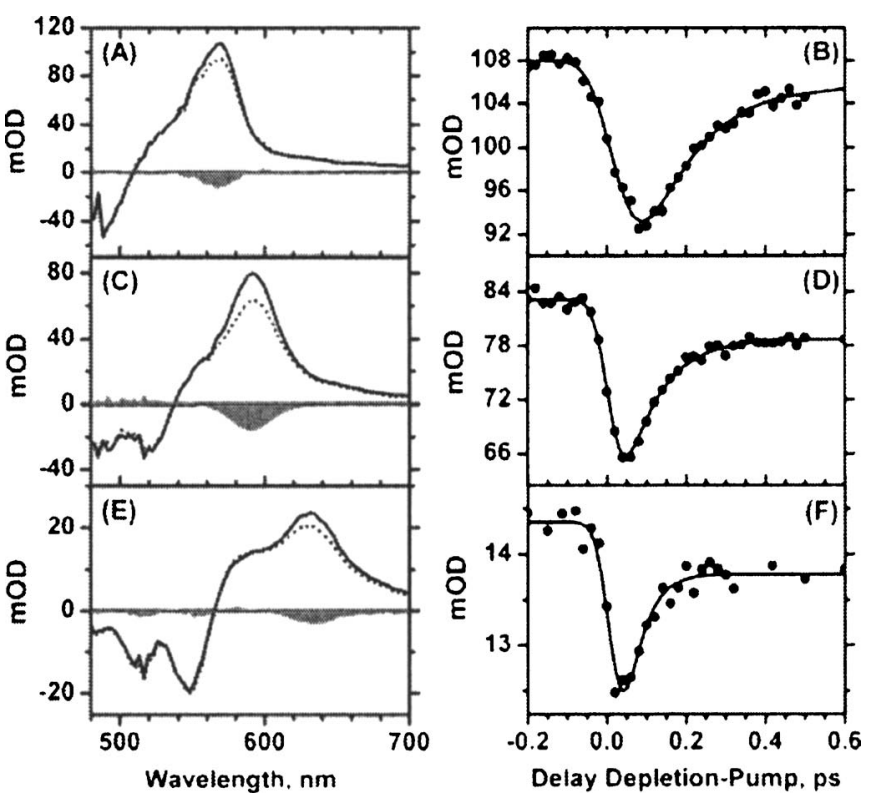

FIG. 3. Depletion spectra probed at 2 ps and depletion transients: first row for M11, second row for M13, and third row for M15. In the depletion transients the probe and the pump were separated by 2 ps. In graphs (A), (C), and (E), the ESA spectra with the depletion pulse present (traced line) and without (continuous line) the depletion pulse are shown. The depletion action spectra were measured for zero delay between the pump and depletion pulses. The difference between them is represented by the gray area. In graphs (B), (D), and (F), the line is a fit with single exponential rise and decay times probed at $570 \mathrm{~nm}$. The depletion pulse was at $1000 \mathrm{~nm}$ in all graphs.

a recovery part described by a single time constant and a plateau, which lives as long as the $S_{1}$ state. For M11 [Fig. $3(\mathrm{~B})]$, the depletion action recovery time showed a time constant of $145 \pm 10$ fs using $1000 \mathrm{~nm}$ which matches those decay times found previously using $795 \mathrm{~nm}$ for depletion. ${ }^{19}$ As can be seen in Fig. 3(A), just the central band of M11 transient absorption is depleted, letting the wing belonging to $S^{*}$ untouched. For the M13 the depletion recovers with a time constant of $90 \pm 10$ fs [Fig. 3(D)], while for the M15 the recovery was about $50 \pm 10$ fs [Fig. $3(\mathrm{~F})]$. When the depletion laser was tuned from 1000 to $940 \mathrm{~nm}$, there was neither a change in dynamics nor in the effect itself for all carotenoids.

\section{DISCUSSION}

The variation of the dynamics of the depletion signal [Figs. 3(B), 3(D), and 3(F)] is compatible with the dynamics of the ultrafast IR bands observed in other experiments. ${ }^{8,13,24}$ In these studies the trend of a systematic decrease of the lifetime of the IR bands with increasing number of conjugated double bonds was observed which corresponds to an equivalent decrease of the depletion duration with increasing number of conjugated double bonds in our experiment. These IR bands were assumed to be initially generated by $S_{2}$ and by dark states $\left(1 B_{u}^{-}\right.$and $\left.3 A_{g}^{-}\right)$few femtoseconds later. In this case, the lifetime of the $S_{2}$ was faster than 10 fs for the carotenoids here studied. Of course, within our instrument response, we cannot observe such time scale unambiguously. However, a 10 fs lifetime for $S_{2}$ would be in disaccord with fluorescence lifetime studies for, e.g., M11, which is in the range of $190-150$ fs. It suggests that indeed the near-IR band absorbing state is the $S_{2}$ state and, therefore, the observed variation of the depletion duration is a manifestation of the $S_{2}$ lifetime variation. This point was recently confirmed by the fluorescence measurement of several carotenoids homologs: ${ }^{25}$ the rise of the depletion action traces matches astonishingly well the observed fluorescence lifetime of the $S_{2}$ state. The lack of a modification on the depletion effect itself and its dynamics when the depletion spectrum was tuned from 940 to $1000 \mathrm{~nm}$ (about $640 \mathrm{~cm}^{-1}$ ), is also compatible with broad bands associated with the near-IR ESA bands, and confirms the homogeneity of the depleted state. Therefore, the whole dynamics can be completely described by the three-level energy model comprising the electronic levels $S_{2}, S_{1}$, and $S_{0}$ and their respective vibrational structures. The assumption of an additional electronically excited state is not necessary with respect to the pump-probe and pump-deplete-probe results as well as to the known fluorescence lifetime, spectrum position, and the $S^{*}$ lifetime. However, an energy level system with an intermediate state with a much faster lifetime than $S_{2}$ would also lead to similar depletion action traces but its relevance with respect to the kinetic network remains probably negligible.

There is still one point that needs to be clarified: the nature of the so called $S^{*}$ state. Its relaxation time does not correspond to the constant of the $S_{1}$ state and does not follow the energy gap rule, i.e., it does not act as an electronically excited state but could rather be interpreted as being part of the electronic ground state. Further indications that $S^{*}$ is a hot ground state were given by the analysis of bleach recovery. In accordance with our previous work, ${ }^{19}$ we assume that $S^{*}$ is a hot ground state populated by impulsive Raman scattering. The ultrashort pump pulse has sufficient bandwidth to contain the Stokes and pump of a Raman excitation. This model is specially appealing since $S^{*}$ is located exactly where the fluorescence from $S_{2}$ should appear, red side of the $S_{0}-S_{2}$ steady-state absorption, which indicates by itself a favorable transition. This mechanism explains why the $S^{*}$ was not depleted: the state is populated instantaneously when the pump is present. $S^{*}$, as a hot ground state, can also be populated through internal conversion from the $S_{1}$ state, possibly leading to the population of other modes than the Ramanactive modes populated by impulsive stimulated Raman scattering (ISRS), which may decay with a different relaxation time.

In the case of the three longer carotenoids, M11, M13, and M15, where the two states $S^{*}$ and $S_{1}$ are spectrally well separated, the lifetime of $S^{*}$ is in the range between 6 and 10 ps. However, the lifetime of $S^{*}$ in the shortest carotenoid, $\mathrm{m} 9$, still remains an open question. If the vibrationally hot ground state is populated from ISRS and from the $S_{1}$, one would expect to observe an additional time constant of about 10 ps in its decay, which was not the case. On the other hand, the analysis of the $S^{*}$ lifetime in $\mathrm{m} 9$ faces some difficulties. Due to its shorter conjugation length, the absorption bands of $S^{*}$ and $S_{1}$ are shifted together towards the blue spectral region and are not well separated anymore. This implies greater difficulty in the disentanglement of the bands and a single wavelength transient might not be sufficient to resemble the dynamics of $S^{*}$. A two-dimensional data set of 

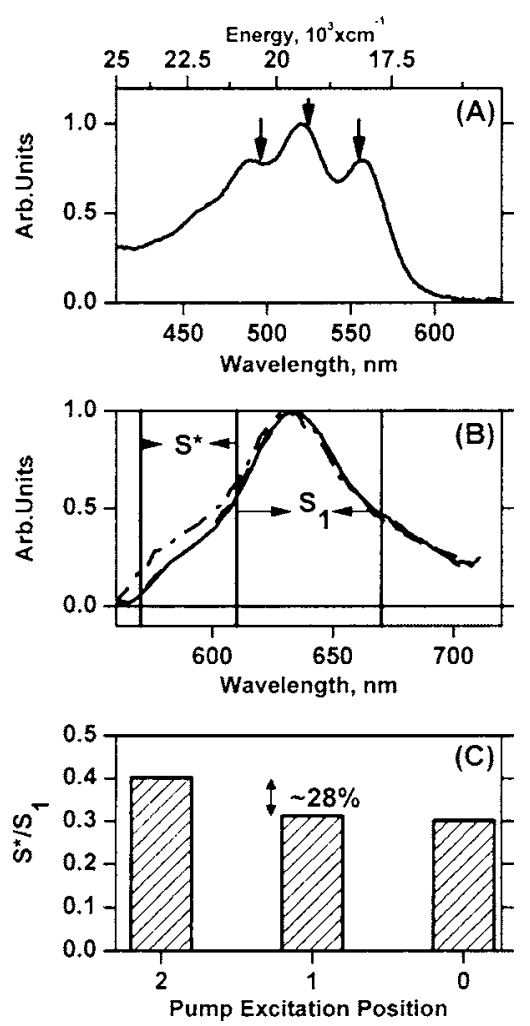

FIG. 4. (A) M15 absorption and the relative position of the pump central wavelength. (B) The maximum ESA spectra for each different excitations: dotted line for 0-0 excitation, traced line for 0-1, and trace-dot line for 0-2. The vertical solid lines indicate the integration limits for what was called $S^{*}$ and $S_{1}$ region. (C) The ratio $S^{*} / S_{1}$ is plotted against the position of pump excitation.

transient broad band spectra in combination with a global target analysis should be able to clearly solve the puzzle of the $S^{*}$ lifetime in $\mathrm{m} 9$.

The hypothesis of $S^{*}$ as a hot ground state is strongly justified if we observe another interesting result when for M15 the pump wavelength was changed from the 0-0 to the 0-2 transition [Fig. 4(A)]. We can compare the $S^{*}$ and $S_{1}$ populations by integrating similar regions where these bands are expected to be. Doing it for comparable bands in the absorption related to the $S^{*}$ (between 570 and $610 \mathrm{~nm}$ ) and $S_{1}$ (between 610 and $670 \mathrm{~nm}$ ) [Fig. 4(B)] and plotting the ratio between the obtained values [Fig. 4(C)], the higher the excitation in $S_{2}$, the more $S^{*}$ we generate. These results can be understood and become much more impressive, if we consider a similar study done for all-trans-neurosporene. ${ }^{27} \mathrm{It}$ was observed that the stimulated emission was strongly affected when the pump excitation was moved from 0-0 to 0-1 in the $S_{0}-S_{2}$ transition. The emission 1-1 could be enhanced and 1-2 decreased when $S_{2}$ was pumped at $v=1$, what is actually just a result of a better Franck-Condon factor, mirroring the displacement of $S_{0}$ and $S_{2}$ potential surfaces. The same is happening here for the M15 when the $S_{2}$ is excited higher: because of a better Franck-Condon in this case, the IRS is greatly enhanced and more $S^{*}$ is populated $(+28 \%)$. The importance of the electronic resonance for IRS in M11 was also recently demonstrated in a coherent control experiment in our group. ${ }^{28}$

\section{CONCLUSION}

The recently found dynamics detected at a spectral range, labeled $S^{*}$, between the ground state bleach and the excited state absorption of $S_{1}$ have been studied by transient absorption and pump-deplete-probe measurements. The pump-depletion-probe shows that the $S^{*}$ band is not affected by the depletion pulse. The analysis of the results leads to the conclusion that all the dynamics can be explained by considering the three well known electronic states $S_{2}, S_{1}$, and $S_{0}$. Besides that, the dynamics of the $S^{*}$ spectral region cannot be brought into agreement with an additional electronic state but can be explained by identifying the results with vibrationally excited ground state dynamics which are instantaneously initiated by impulsive Raman scattering. In addition to the fact that the assumed new electronic state $S^{*}$ does not obey the energy gap rule for electronically excited states, as, e.g., the $S_{1}$ does, the application of pump-deplete-probe experiments proved the different natures of the two states. After depletion of $S_{2}$, only the $S_{1}$ population was modified but the $S^{*}$ population remained unchanged and therefore cannot depend on the deactivation pathway from $S_{2}$. Although the standard transient absorption results cannot exclude the existence of more participating dark states below the $S_{2}$ state, the pump-depletion-probe experiment clearly elucidates the near-infrared dynamics which can be explained by a band that rises very fast and decays with times from 50 to $150 \mathrm{fs}$.

\section{ACKNOWLEDGMENTS}

Generous support by Karl Kompa and the Max-PlanckSociety is gratefully appreciated. One of the authors (T.B.) acknowledges the grant from CNPq (Brasil). Support from the ESF is also acknowledged.

${ }^{1}$ T. Polivka and V. Sundstrom, Chem. Rev. (Washington, D.C.) 104, 2021 (2004).

${ }^{2}$ R. J. Cogdell, P. K. Fyfe, T. D. Howard, N. J. Fraser, N. W. Isaacs, A. A. Freer, K. McKluskey, and S. M. Prince, in The Photochemistry of Carotenoids, edited by H. A. Frank, A. J. Young, G. Britton, and R. J. Cogdell (Kluwer Academic, Dordrecht, 1999), p. 71.

${ }^{3}$ R. L. Christensen, in The Photochemistry of Carotenoids (Ref. 2), p. 137.

${ }^{4}$ M. Yoshizawa, H. Aoki, and H. Hashimoto, Phys. Rev. B 6318, 180301 (2001).

${ }^{5}$ M. Yoshizawa, H. Aoki, M. Ue, and H. Hashimoto, Phys. Rev. B 67, 174302 (2003).

${ }^{6}$ H. H. Billsten, D. Zigmantas, V. Sundstrom, and T. Polivka, Chem. Phys. Lett. 355, 465 (2002).

${ }^{7}$ R. Englman and J. Jortner, Mol. Phys. 18, 145 (1970).

${ }^{8}$ R. Fujii, T. Inaba, Y. Watanabe, Y. Koyama, and J. P. Zhang, Chem. Phys. Lett. 369, 165 (2003).

${ }^{9}$ M. Ikuta, A. Yabushita, F. S. Rondonuwu, J. Akahane, Y. Koyama, and T. Kobayashi, Chem. Phys. Lett. 422, 95 (2006).

${ }^{10}$ T. Sashima, H. Nagae, M. Kuki, and Y. Koyama, Chem. Phys. Lett. 299, 187 (1999).

${ }^{11}$ T. Sashima, Y. Koyama, T. Yamada, and H. Hashimoto, J. Phys. Chem. B 104, 5011 (2000).

${ }^{12}$ K. Furuichi, T. Sashima, and Y. Koyama, Chem. Phys. Lett. 356, 547 (2002).

${ }^{13}$ G. Cerullo, D. Polli, G. Lanzani, S. De Silvestri, H. Hashimoto, and R. J. Cogdell, Science 298, 2395 (2002).

${ }^{14}$ D. Kosumi, M. Komukai, H. Hashimoto, and M. Yoshizawa, Phys. Rev. Lett. 95, 213601 (2005).

${ }^{15}$ C. C. Gradinaru, J. T. M. Kennis, E. Papagiannakis, I. H. M. van Stokkum, R. J. Cogdell, G. R. Fleming, R. A. Niederman, and R. van Grondelle, Proc. Natl. Acad. Sci. U.S.A. 98, 2364 (2001).

${ }^{16}$ W. Wohlleben, T. Buckup, J. L. Herek, R. J. Cogdell, and M. Motzkus, 
Biophys. J. 85, 442 (2003).

${ }^{17}$ P. O. Andersson and T. Gillbro, J. Chem. Phys. 103, 2509 (1995).

${ }^{18}$ E. Papagiannakis, I. H. M. van Stokkum, M. Vengris, R. J. Cogdell, R. van Grondelle, and D. S. Larsen, J. Phys. Chem. B 110, 5727 (2006).

${ }^{19}$ W. Wohlleben, T. Buckup, H. Hashimoto, R. J. Cogdell, J. L. Herek, and M. Motzkus, J. Phys. Chem. B 108, 3320 (2004).

${ }^{20}$ F. Gai, J. C. McDonald, and P. A. Anfinrud, J. Am. Chem. Soc. 119, 6201 (1997).

${ }^{21}$ M. Yan, L. Rothberg, and R. Callender, J. Phys. Chem. B 105, 856 (2001).

${ }^{22}$ D. S. Larsen, E. Papagiannakis, I. H. M. van Stokkum, M. Vengris, J. T. M. Kennis, and R. van Grondelle, Chem. Phys. Lett. 381, 733 (2003).

${ }^{23}$ A. N. Macpherson and T. Gillbro, J. Phys. Chem. A 102, 5049 (1998).
${ }^{24}$ D. Polli, G. Cerullo, G. Lanzani, S. De Silvestri, K. Yanagi, H. Hashimoto, and R. J. Cogdell, Phys. Rev. Lett. 93, 163002 (2004).

${ }^{25}$ D. Kosumi, K. Yanagi, R. Fujii, H. Hashimoto, and M. Yoshizawa, Chem. Phys. Lett. 425, 66 (2006).

${ }^{26}$ S. Akimoto, I. Yamazaki, S. Takaishi, and M. Mimuro, Chem. Phys. Lett. 313, 63 (1999).

${ }^{27}$ J.-P. Zhang, T. Inaba, Y. Watanabe, and Y. Koyama, Chem. Phys. Lett. 331, 154 (2000).

${ }^{28}$ J. Hauer, H. Skenderovic, K. L. Kompa, and M. Motzkus, Chem. Phys. Lett. 421, 523 (2006).

${ }^{29}$ D. Polli, G. Cerullo, G. Lanzani, S. De Silvestri, H. Hashimoto, and R. J. Cogdell, Synth. Met. 139, 893 (2003). 